\title{
Physiologic specialization of Puccinia triticina Erikss. and effectiveness of Lr-genes in the south of Ukraine during 2013-2014
}

\author{
Olga Babayants', Lazar Babayants ${ }^{1}$ Andrii Gorash²*, Alexey Vasilev¹, Vita Traskovetskaya1, \\ and Alexey Galaev ${ }^{1}$
}

\begin{abstract}
Leaf rust is the most widespread and frequently occurring fungal disease of wheat (Triticum aestivum L.) in Ukraine and worldwide. The information about the effectiveness of $L r$-genes and also the consequent monitoring of virulence dynamics is necessary for the successful wheat breeding for leaf rust resistance. In 2013-2014 pathotype composition and virulence analysis was studied both on the standard differential set and on the North American System of Nomenclature. According to the standard differential set, 12 phenotypes were identified, of which the most common were $77(75 \%)$ and $144(6 \%)$. A total of 40 phenotypes were identified on the North American Nomenclature. Phenotypes TGTT (24\%) and TJTT (8\%) were the most frequent, TRTT $(1.5 \%)$ and TSTT $(1.5 \%)$ were within the broadest spectrum of virulence among the isolates found in the south of Ukraine. For virulence analysis we used wheat lines of 'Thatcher' that are near-isogenic for 24 leaf rust resistance genes and additionally four cultivars/lines. No virulence to $L r 19$ was found, whereas increasing virulence to $\operatorname{Lr} 9$ was detected (13\%). Low frequency of virulence was observed to $\operatorname{Lr} 29$ (11\%) and $\operatorname{Lr} 47$ (21\%), high level of virulence was detected to other genes. The effectiveness of 53 known $L r$-genes was studied at the seedling and the adult plant stages. Most of them were not effective against leaf rust. Genes $\operatorname{Lr} 9, \operatorname{Lr} 19, \operatorname{Lr} 29$, and $\operatorname{Lr} 47$ were highly effective both at the seedling stage and at adult plant stage. Genes $L r 24, \operatorname{Lr} 42, \operatorname{Lr} 50, \operatorname{Lr} 51$, and $L r 56$ were effective only at the adult plant stage.
\end{abstract}

Key words: Leaf rust, resistance, Triticum aestivum, virulence analysis, wheat.

\section{INTRODUCTION}

Leaf rust (caused by Puccinia triticina Erikss.) is the most widespread and frequently occurring fungal disease of wheat (Triticum aestivum L.) in Ukraine and throughout the world (Babayants, 2011; Huerta-Espino et al., 2011). Growing of resistantcultivars is the safest and economically profitable way to control fungal plant diseases. However, wheat breeding for resistance is complicated because of the pathogen's ability to overcome the host resistance. The new pathotypes are continuously emerging due to sexual recombination and mutational processes. The nature of urediniospores enables them to migrate by air for thousands of kilometers, which causes the spread of new virulent pathotypes throughout the world (Kolmer, 2005). The information about the effectiveness of $L r$-genes and also the consequent monitoring of virulence dynamics are necessary for successful wheat breeding for resistance to

${ }^{1}$ Plant Breeding and Genetics Institute, National Center of Seed and Cultivar Investigation, 65036, Odessa, Ukraine.

${ }^{2}$ Institute of Agriculture, Lithuanian Research Centre for Agriculture and Forestry, Department of Cereal Breeding, LT-58344, Akademija, Kèdainiai distr., Lithuania. "Corresponding author (andrej@1zi.lt).

Received: 19 December 2014

Accepted: 21 May 2015

doi:10.4067/S0718-58392015000500009 leaf rust and for timely detection of new phenotypes to adjust the breeding programs.

A nomenclature to divide the population of leaf rust into separate pathotype "races" was proposed after discovery of physiologic races by Mains and Jackson in 1926 (Mains and Jackson, 1926). Then, after a few modifications, the nomenclature was adopted on the basis of eight cultivars (Johnson and Browder, 1966). However, after emergence of new pathotypes which, according to their characteristics, did not match up with this code, different researchers assigned them different designations. Another disadvantage is that these differentials carry $L r$-genes in different genetic background and some of them carry several $L r$-genes, for example 'Carina' ( $L r 2 b, L r B)$, 'Brevit' ( $L r 2 c$, LrB ), 'Webster' (Lr2a, Lr14a, Lr27), 'Loros' (Lr2c, $L r 2 d)$, 'Mediterranean' (Lr2a, Lr3a) share different Lr-genes (McIntosh et al., 1995; GRIS, 2014). When the near-isogenic lines which possess single $L r$-genes were developed they started to be used as differentials among new set of wheat cultivars. Most researchers have replaced the term physiologic race by the term pathotype or phenotype (Kolmer, 2005; Hanzalova and Bartos, 2006). In 1989, a North American System of Nomenclature for Puccinia triticina was proposed. According to this differential set, it was proposed to use 12 near-isogenic lines of 'Thatcher' with single $L r$ - 
genes. The differentials were grouped into three sets of four lines. The first set of differentials consisted of 'Thatcher' lines with genes $L r 1, L r 2 a, L r 2 c, L r 3 a$, the second Lr9, Lr16, Lr24, Lr26, the third Lr3ka, Lr11, $L r 17 a, L r 30$. The pathotypes characteristics are coded in accordance with the type of reaction by one of 16 letters for each of three sets; accordingly, a three-letter code was assigned to the pathotypes (Long and Kolmer, 1989). This nomenclature has become the most common and is being extensively used throughout most of the world (Huerta-Espino et al., 2011). Virulence analysis of leaf rust population requires information about changes of virulence first of all for genes which present interest for specific climatic-territorial areas where the cultivars are planned to be grown. Therefore phytopathologists are attempting to add the differentials with certain $\mathrm{Lr}$ genes to the differential set, according to specificity of pathogen's population of their region. In the last years, a series of additional sets of 'Thatcher' near-isogenic lines has been proposed to be included in the original North American differential set in the USA and other countries (Singh, 1991; Kolmer and Liu, 2000; McVey et al., 2004; Mantovani et al., 2010). Supplemental fourth set of $\operatorname{LrB}, \mathrm{Lr} 10, \mathrm{Lr} 14 \mathrm{a}$, and $\mathrm{Lr} 18$ was added by Kolmer et al. (2007) and included in the national virulence surveys in the USA and Canada. The fifth set of differentials with two 'Thatcher' lines with $\operatorname{Lr} 21, \operatorname{Lr} 28$ and two winter wheat lines with genes $L r 41$ and $L r 42$ were added to the national virulence surveys in the USA in 2004, but in 2007 the line with $\operatorname{Lr} 42$ was dropped after it had been determined that it also possessed Lr24 (Kolmer et al., 2007; Kolmer and Hughes, 2013).

In European countries, a standard differential set which consists of 15 Thatcher near isogenic lines with the single leaf rust genes $L r 1, L r 2 a, L r 2 b, L r 2 c, L r 3 a, L r 9, L r 11$, Lr15, Lr17, Lr19, Lr21, Lr23, Lr24, Lr26 and Lr28 has been adopted. Pathotype analysis is conducted using digital nomenclature with triplet set and octal codes of virulence according to Hanzalova and Bartos (2006) and to Goyeau et al. (2006). Researchers in Nepal, Bangladesh, Pakistan, South Africa, and others countries have reported races using both their own nomenclatures and the North American Nomenclature. However, breeders from India and Australia are still using their own binomial systems of nomenclature (Huerta-Espino et al., 2011; Terefe et al., 2014).

Physiologic races of $P$. triticina have been studied in Plant Breeding and Genetics Institute in Ukraine since the 1960 's on an old standard differential set. In the present study, we used both the old standard differential set and the North American Nomenclature to compare the old findings of the old standard differential set with the North American differential set for $P$. triticina. The aim of the study was to conduct a pathotype analysis of leaf rust population and to study the effectiveness of 53 known $\mathrm{Lr}$ genes at the seedling and adult plant stages in the south of Ukraine.

\section{MATERIAL AND METHODS}

Samples of leaf rust infected wheat leaves of different cultivars and breeding lines were collected from commercial fields and research plots at various locations throughout the south of Ukraine. All collected leaves were air dried at room temperature and stored at $4{ }^{\circ} \mathrm{C}$ until spores were collected for inoculation and multiplication of the inoculum. The virulence survey was based on monopustule isolates. Puccinia triticina urediniospores from individual collections were scraped off and mixed with water and Tween 20. The susceptible cultivars 'Michigan Amber' and 'Odesskaya polukarlikovaya' were inoculated with the suspension of urediniospores. Approximately $14 \mathrm{~d}$ after inoculation, using a microbiological loop, separate monopustule isolates were transferred on the detached leaves of 'Michigan Amber' and 'Odesskaya polukarlikovaya', kept in the solution of benzimidazol in Petri dishes (Babayants and Babayants, 2014). Microbiological loop was sterilized each time before transferring the new pustules. Each monopustule isolate was transferred into a separate Petri dish. When the inoculum was multiplied, approximately after 14 $\mathrm{d}$, differentials were also inoculated using the same procedure.

A total of 113 monopustule isolates for phenotype and virulence analyses were studied. Phenotype analysis was conducted on the standard differential set of eight cultivars: Malakoff, Carina, Brevit, Webster, Loros, Mediterranean, Hussar, Democrat (Johnson and Browder, 1966) and the North American differential set for $P$. triticina on 16 near-isogenic lines of 'Thatcher' with single leaf rust resistance genes $L r 1, L r 2 a, L r 2 c, L r 3 a$, Lr3ka, Lr9, Lr10, Lr11, Lr14a, Lr16, Lr17a, Lr18, Lr24, Lr26, Lr30, and LrB (Long and Kolmer, 1989; Kolmer et al., 2007).

Virulence analysis of leaf rust population was studied on the near-isogenic lines of 'Thatcher' with single genes: LrB, Lr1, Lr2a, Lr2c, Lr3a, Lr3ka, Lr9, Lr10, Lr11, Lr14a, Lr17, Lr18, Lr19, Lr20, Lr21, Lr22a, Lr23, Lr24, Lr25, Lr26, Lr29, Lr30, Lr38, and Lr64. In addition four cultivars/lines 'Pavon 76' (Lr1, Lr10, Lr13, Lr14a, Lr2b, Lr22a, Lr27, Lr46), 'Pavon 753' (Lr47), 'KS86WGRC02' (Lr39) and 'KS92WGRC16' (Lr42, Lr39, Lr21) were used.

The effectiveness of $53 \mathrm{Lr}$-genes was studied both at the seedling stage and at the stage of adult plant. The resistance at the seedling stage was assessed in the greenhouse under controlled conditions (temperature: $+20 \pm 2{ }^{\circ} \mathrm{C}$, illuminance: $10000 \mathrm{lux}, 16: 8 \mathrm{~h}$ photoperiod). Ten days old plants were inoculated by a mix of urediniospores with talcum powder. After inoculation, to create the conditions of a dew chamber, the plants were put in the polyethylene bags and stored in the dark at 21 ${ }^{\circ} \mathrm{C}$ for $16 \mathrm{~h}$. Type of resistance and disease severity were scored on the $12^{\text {th }}$ day after inoculation. Type of resistance 
at the seedling stage was scored according to the scale: very resistant (VR), resistant (R), moderately resistant (MR), moderately susceptible (MS), susceptible (S), and very susceptible (VS). Disease severity was scored by the scale of Peterson (Babayants and Babayants, 2014).

The field resistance was studied in the artificially infected nursery. The differentials were grown in $1 \mathrm{~m}$ long rows (three rows for each differential), the distance between rows was $30 \mathrm{~cm}$. Two rows of the most rustsusceptible spreader ('Odesskaya polukarlikovaya') were planted around the nursery. Also, one row of this susceptible control was planted at every $10^{\text {th }}$ test entry. The leaf rust differentials were inoculated with a mixture of urediniospores and talcum powder according to Babayants procedure (Babayants and Babayants, 2014). Inoculation was done in 22 April 2013 and 28 April 2014. Environmental conditions for pathogen development are presented in Table 1. Adult resistance in the field was scored according to a 9 point scale: 1-2 very high susceptibility, 3 high susceptibility, 4 susceptibility,

Table 1. Meteorological data during the inoculation and Puccinia triticina development.

\begin{tabular}{|c|c|c|c|c|c|c|}
\hline \multirow{2}{*}{$\begin{array}{l}\text { Year, } \\
\text { Month } \\
\end{array}$} & \multirow[b]{2}{*}{ Decade } & \multirow[b]{2}{*}{ Precipitation } & \multirow{2}{*}{$\begin{array}{l}\text { Relative } \\
\text { humidity }\end{array}$} & \multicolumn{3}{|c|}{ Air temperature } \\
\hline & & & & Max & Min & Avg \\
\hline & & $\mathrm{mm}$ & $\%$ & & ${ }^{\circ} \mathrm{C}$ & \\
\hline \multicolumn{7}{|l|}{2013} \\
\hline & 1 & 2.3 & 80 & 22.2 & 2.5 & 8.0 \\
\hline \multirow[t]{3}{*}{ April } & 2 & 36.1 & 75 & 22.3 & 1.8 & 9.8 \\
\hline & 3 & 0.7 & 64 & 27.6 & 5.0 & 15.7 \\
\hline & 1 & 0.0 & 68 & 29.2 & 11.5 & 18.7 \\
\hline \multirow[t]{3}{*}{ May } & 2 & 4.0 & 77 & 28.7 & 11.4 & 19.2 \\
\hline & 3 & 0.8 & 81 & 29.4 & 11.5 & 19.3 \\
\hline & 1 & 29.1 & 84 & 28.0 & 12.0 & 19.0 \\
\hline \multirow[t]{2}{*}{ June } & 2 & 54.4 & 70 & 32.0 & 14.9 & 22.8 \\
\hline & 3 & 41.2 & 73 & 34.2 & 14.3 & 24.0 \\
\hline \multicolumn{7}{|l|}{2014} \\
\hline & 1 & - & 68 & 22.6 & -2.3 & 8.6 \\
\hline \multirow[t]{3}{*}{ April } & 2 & 4.6 & 82 & 23.6 & 5.0 & 10.8 \\
\hline & 3 & - & 70 & 23.4 & 5.3 & 13.9 \\
\hline & 1 & 9.8 & 75 & 22.7 & 3.3 & 13.7 \\
\hline \multirow[t]{3}{*}{ May } & 2 & 11.6 & 78 & 25.7 & 9.9 & 16.3 \\
\hline & 3 & 4.8 & 69 & 32.7 & 13.3 & 21.3 \\
\hline & 1 & 46.1 & 67 & 34.0 & 10.3 & 21.6 \\
\hline \multirow[t]{2}{*}{ June } & 2 & 14.0 & 62 & 30.0 & 12.3 & 20.7 \\
\hline & 3 & 7.5 & 69 & 29.0 & 10.4 & 20.0 \\
\hline
\end{tabular}

Max: maximum, Min: minimum, Avg: average.
5 moderate susceptibility, 6 moderate resistance, 7 resistance, 8 high resistance, and 9 immunity (Babayants and Babayants, 2014). The seeds of differentials and wheat lines/cultivars with $L r$-genes were provided via USDA Germplasm Resources Information Network (GRIN) (USDA ARS, 2014).

\section{RESULTS}

\section{Phenotype composition}

Phenotype analysis was conducted using two differential sets - the standard differential set and the North American System of Nomenclature for P. triticina. On the standard differential set, 12 different phenotypes were revealed in 2013. The most common races were $77(75 \%)$ and 144 (6\%), noticed also the others $149(4 \%), 117(3 \%), 192$ (2\%), $114(2 \%), 6(2 \%), 21(2 \%), 42(1 \%), 57(1 \%), 20$ (1\%), 122 (1\%) (Table 2). Forty phenotypes for P. triticina were identified on the North American Nomenclature. Among them two phenotypes TGTT (24\%) and TJTT (8\%) were dominant. Phenotypes PHTT, TQTT, TGPT, RGTT, TGKT, and PBKK had an occurrence frequency of $3 \%$ each, while others were represented by single isolates. Phenotype TGTT was avirulent to $\operatorname{Lr} 9, \operatorname{Lr} 24$ and Lr26 and it was virulent against the other $13 \mathrm{Lr}$-genes of the differential set. TJTT was avirulent only to $\operatorname{Lr} 9$ and Lr26. Phenotypes TRTT and TSTT with the frequency of occurrence of $1.5 \%$ possess the broadest spectrum of virulence, the former was avirulent only to $\operatorname{Lr} 24$ and the latter to $L r 26$. A total of 40 phenotypes of wheat leaf rust were identified on the North America Nomenclature in the south of Ukraine in 2013 (Table 3).

\section{Virulence analysis of Puccinia triticina population}

The virulence analysis was done on 26 near isogenic lines of 'Thatcher' and also on the four wheat cultivars/lines (Tables 4 and 5). None of the 113 studied monopustule isolates had virulence to gene $\operatorname{Lr} 19$. In the previous years the frequency of virulence to $\operatorname{Lr} 19$ varied from $0 \%$ to $4 \%$ (Babayants et al., 2004; Babayants, 2011).

Table 2. The virulence formula and frequency of Puccinia triticina phenotypes identified on the standard differential set in the south of Ukraine in 2013.

\begin{tabular}{|c|c|c|c|c|c|c|c|c|c|}
\hline Race & Malakof & Carina & Brevit & Webster & Loros & Mediterranean & Hussar & Democrat & $\%$ \\
\hline 77 & $\mathrm{~S}$ & $\mathrm{~S}$ & $\mathrm{~S}$ & $\mathrm{~S}$ & $\mathrm{~S}$ & $\mathrm{~S}$ & $\mathrm{~S}$ & $\mathrm{~S}$ & 75 \\
\hline 144 & $\mathrm{~S}$ & $\mathrm{~S}$ & $\mathrm{~S}$ & $\mathrm{R}$ & $\mathrm{S}$ & $\mathrm{S}$ & $\mathrm{S}$ & $\mathrm{S}$ & 6 \\
\hline 149 & $\mathrm{~S}$ & $\mathrm{R}$ & $\mathrm{S}$ & $\mathrm{S}$ & $\mathrm{S}$ & $\mathrm{S}$ & $\mathrm{S}$ & $\mathrm{S}$ & 4 \\
\hline 117 & $\mathrm{~S}$ & $\mathrm{~S}$ & $\mathrm{~S}$ & $\mathrm{~S}$ & $\mathrm{~S}$ & $\mathrm{R}$ & $\mathrm{S}$ & $\mathrm{S}$ & 3 \\
\hline 192 & $\mathrm{~S}$ & $\mathrm{~S}$ & $\mathrm{~S}$ & $\mathrm{~S}$ & $\mathrm{R}$ & $\mathrm{S}$ & $\mathrm{S}$ & $\mathrm{S}$ & 2 \\
\hline 114 & $\mathrm{~S}$ & $\mathrm{~S}$ & $\mathrm{~S}$ & I & $\mathrm{S}$ & $\mathrm{S}$ & $\mathrm{R}$ & $\mathrm{S}$ & 2 \\
\hline 6 & $\mathrm{~S}$ & $\mathrm{R}$ & $\mathrm{S}$ & $\mathrm{R}$ & $\mathrm{S}$ & $\mathrm{S}$ & $\mathrm{S}$ & $\mathrm{S}$ & 2 \\
\hline 21 & $\mathrm{~S}$ & $\mathrm{~S}$ & $\mathrm{R}$ & $\mathrm{S}$ & $\mathrm{S}$ & $\mathrm{S}$ & $\mathrm{S}$ & $\mathrm{S}$ & 2 \\
\hline 42 & $\mathrm{~S}$ & I & $\mathrm{S}$ & $\mathrm{S}$ & $\mathrm{S}$ & $\mathrm{S}$ & $\mathrm{S}$ & $\mathrm{S}$ & 1 \\
\hline 57 & $\mathrm{R}$ & $\mathrm{S}$ & $\mathrm{S}$ & $\mathrm{S}$ & $\mathrm{S}$ & $\mathrm{S}$ & $\mathrm{S}$ & $\mathrm{S}$ & 1 \\
\hline 122 & $\mathrm{~S}$ & $\mathrm{~S}$ & $\mathrm{~S}$ & $\mathrm{~S}$ & $\mathrm{~S}$ & $\mathrm{~S}$ & $\mathrm{R}$ & $\mathrm{S}$ & 1 \\
\hline 20 & $\mathrm{~S}$ & $\mathrm{~S}$ & $\mathrm{~S}$ & $\mathrm{~S}$ & $\mathrm{~S}$ & $\mathrm{R}$ & $\mathrm{S}$ & $\mathrm{R}$ & 1 \\
\hline Total & & & & & & & & & 100 \\
\hline
\end{tabular}

R: Resistant, S: susceptible, I: intermediate. 
Table 3. Virulence formula and frequency of Puccinia triticina phenotypes in the south of Ukraine in 2013 identified on the North American Nomenclature (16 near-isogenic lines with single $L r$-genes).

\begin{tabular}{|c|c|c|c|}
\hline Phenotype & Avirulence/virulence formula & Pcs. & $\%$ \\
\hline TGTT & Lr9, Lr24, Lr26/Lr1, Lr2a, Lr2c, Lr3a, Lr16, Lr3ka, Lr11, Lr17a, Lr30, LrB, Lr10, Lr14a, Lr18 & 16 & 24 \\
\hline TJTT & Lr9, Lr26/Lr1, Lr2a, Lr2c, Lr3a, Lr16, Lr24, Lr3ka, Lr11, Lr17a, Lr30, LrB, Lr10, Lr14a, Lr18 & 5 & 8 \\
\hline PHTT & Lr2a, Lr9, Lr24/Lr1, Lr2c, Lr3a, Lr16, Lr26, Lr3ka, Lr11, Lr17a, Lr30, LrB, Lr10, Lr14a, Lr18 & 2 & 3 \\
\hline TQTT & Lr24, Lr26/Lr1, Lr2a, Lr2c, Lr3a, Lr9, Lr16, Lr3ka, Lr11, Lr17a, Lr30, LrB, Lr10, Lr14a, Lr18 & 2 & 3 \\
\hline TGPT & Lr9, Lr24, Lr11/Lr1, Lr2a, Lr2c, Lr3a, Lr16, Lr26, Lr3ka, Lr17a, Lr30, LrB, Lr10, Lr14a, Lr18 & 2 & 3 \\
\hline TGKT & Lr9, Lr24, Lr26, Lr3ka/Lr1, Lr2a, Lr2c, Lr3a, Lr16, Lr11, Lr17a, Lr30, LrB, Lr10, Lr14a, Lr18 & 2 & 3 \\
\hline RJTT & Lr2c, Lr9, Lr26/Lr1, Lr2a, Lr3a, Lr16, Lr24, Lr3ka, Lr11, Lr17a, Lr30, LrB, Lr10, Lr14a, Lr18 & 2 & 3 \\
\hline RGTT & Lr2c, Lr9, Lr24, Lr26/Lr1, Lr3a, Lr16, Lr3ka, Lr11, Lr17a, Lr30, LrB, Lr10, Lr14a, Lr18 & 2 & 3 \\
\hline PBKK & Lr2a, Lr9, Lr16, Lr24, Lr26, Lr3ka, LrB/Lr1, Lr2c, Lr3a, Lr11, Lr17a, Lr30, Lr10, Lr14a, Lr18 & 2 & 3 \\
\hline TRTT & Lr24/Lr1, Lr2a, Lr2c, Lr3a, Lr9, Lr16, Lr26, Lr3ka, Lr11, Lr17a, Lr30, LrB, Lr10, Lr14a, Lr18 & 1 & 1.5 \\
\hline TSTT & Lr26/Lr1, Lr2a, Lr2c, Lr3a, Lr9, Lr16, Lr24, Lr3ka, Lr11, Lr17a, Lr30, LrB, Lr10, Lr14a, Lr18 & 1 & 1.5 \\
\hline THTT & Lr9, Lr24/Lr1, Lr2a, Lr2c, Lr3a, Lr16, Lr26, Lr3ka, Lr11, Lr17a, Lr30, LrB, Lr10, Lr14a, Lr18 & 1 & 1.5 \\
\hline TQSP & Lr24, Lr26, Lr30, Lr10/Lr1, Lr2a, Lr2c, Lr3a, Lr9, Lr16, Lr3ka, Lr11, Lr17a, LrB, Lr14a, Lr18 & 1 & 1.5 \\
\hline TQQN & Lr24, Lr26, Lr17a, Lr30, Lr10, Lr18/Lr1, Lr2a, Lr2c, Lr3a, Lr9, Lr16, Lr3ka, Lr11, LrB, Lr14a & 1 & 1.5 \\
\hline TNTT & Lr16, Lr26/Lr1, Lr2a, Lr2c, Lr3a, Lr9, Lr24, Lr3ka, Lr11, Lr17a, Lr30, LrB, Lr10, Lr14a, Lr18 & 1 & 1.5 \\
\hline TJKT & Lr9, Lr26, Lr3ka/Lr1, Lr2a, Lr2c, Lr3a, Lr16, Lr24, Lr11, Lr17a, Lr30, LrB, Lr10, Lr14a, Lr18 & 1 & 1.5 \\
\hline TGTM & Lr9, Lr24, Lr26, Lr10, Lr14a/Lr1, Lr2a, Lr2c, Lr3a, Lr16, Lr3ka, Lr11, Lr17a, Lr30, LrB, Lr18 & 1 & 1.5 \\
\hline TGQM & Lr9, Lr24, Lr26, Lr17a, Lr30, Lr10, Lr14a/Lr1, Lr2a, Lr2c, Lr3a, Lr16, Lr3ka, Lr11, LrB, Lr18 & 1 & 1.5 \\
\hline TGQT & Lr9, Lr24, Lr26, Lr17a, Lr30/Lr1, Lr2a, Lr2c, Lr3a, Lr16, Lr3ka, Lr11, LrB, Lr10, Lr14a, Lr18 & 1 & 1.5 \\
\hline TDQL & Lr9, Lr16, Lr26, Lr17a, Lr30, Lr10, Lr14a, Lr18/Lr1, Lr2a, Lr2c, Lr3a, Lr24, Lr3ka, Lr11, LrB & 1 & 1.5 \\
\hline TBTT & Lr9, Lr16, Lr24, Lr26/Lr1, Lr2a, Lr2c, Lr3a, Lr3ka, Lr11, Lr17a, Lr30, LrB, Lr10, Lr14a, Lr18 & 1 & 1.5 \\
\hline RGHT & Lr2c, Lr9, Lr24, Lr26, Lr3ka, Lr17a/Lr1, Lr2a, Lr3a, Lr16, Lr11, Lr30, LrB, Lr10, Lr14a, Lr18 & 1 & 1.5 \\
\hline RHTT & Lr2c, Lr9, Lr24/Lr1, Lr2a, Lr3a, Lr16, Lr26, Lr3ka, Lr11, Lr17a, Lr30, LrB, Lr10, Lr14a, Lr18 & 1 & 1.5 \\
\hline RKTT & Lr2c, Lr9/Lr1, Lr2a, Lr3a, Lr16, Lr24, Lr26, Lr3ka, Lr11, Lr17a, Lr30, LrB, Lr10, Lr14a, Lr18 & 1 & 1.5 \\
\hline QGMS & Lr2c, Lr3a, Lr9, Lr24, Lr26, Lr11, Lr17a, Lr18/Lr1, Lr2a, Lr16, Lr3ka, Lr30, LrB, Lr10, Lr14a & 1 & 1.5 \\
\hline PQFT & Lr2a, Lr24, Lr26, Lr3ka, Lr11/Lr1, Lr2c, Lr3a, Lr9, Lr16, Lr17a, Lr30, LrB, Lr10, Lr14a, Lr18 & 1 & 1.5 \\
\hline PKTT & Lr2a, Lr9/Lr1, Lr2c, Lr3a, Lr16, Lr24, Lr26, Lr3ka, Lr11, Lr17a, Lr30, LrB, Lr10, Lr14a, Lr18 & 1 & 1.5 \\
\hline PJTT & Lr2a, Lr9, Lr26/Lr1, Lr2c, Lr3a, Lr16, Lr24, Lr3ka, Lr11, Lr17a, Lr30, LrB, Lr10, Lr14a, Lr18 & 1 & 1.5 \\
\hline PHCT & Lr2a, Lr9, Lr24, Lr3ka, Lr11, Lr17a/Lr1, Lr2c, Lr3a, Lr16, Lr26, Lr30, LrB, Lr10, Lr14a, Lr18 & 1 & 1.5 \\
\hline PGTT & Lr2a, Lr9, Lr24, Lr26/Lr1, Lr2c, Lr3a, Lr16, Lr3ka, Lr11, Lr17a, Lr30, LrB, Lr10, Lr14a, Lr18 & 1 & 1.5 \\
\hline PGFT & Lr2a, Lr9, Lr24, Lr26, Lr3ka, Lr11/Lr1, Lr2c, Lr3a, Lr16, Lr17a, Lr30, LrB, Lr10, Lr14a, Lr18 & 1 & 1.5 \\
\hline NGCS & Lr2a, Lr3a, Lr9, Lr24, Lr26, Lr3ka, Lr11, Lr17a, Lr18/Lr1, Lr2c, Lr16, Lr30, LrB, Lr10, Lr14a & 1 & 1.5 \\
\hline NGKT & Lr2a, Lr3a, Lr9, Lr24, Lr26, Lr3ka/Lr1, Lr2c, Lr16, Lr11, Lr17a, Lr30, LrB, Lr10, Lr14a, Lr18 & 1 & 1.5 \\
\hline NHTT & Lr2a, Lr3a, Lr9, Lr24/Lr1, Lr2c, Lr16, Lr26, Lr3ka, Lr11, Lr17a, Lr30, LrB, Lr10, Lr14a, Lr18 & 1 & 1.5 \\
\hline KSTT & Lr1, Lr26/Lr2a, Lr2c, Lr3a, Lr9, Lr16, Lr24, Lr3ka, Lr11, Lr17a, Lr30, LrB, Lr10, Lr14a, Lr18 & 1 & 1.5 \\
\hline KJTT & Lr1, Lr9, Lr26/Lr2a, Lr2c, Lr3a, Lr16, Lr24, Lr3ka, Lr11, Lr17a, Lr30, LrB, Lr10, Lr14a, Lr18 & 1 & 1.5 \\
\hline KGTT & Lr1, Lr9, Lr24, Lr26/Lr2a, Lr2c, Lr3a, Lr16, Lr3ka, Lr11, Lr17a, Lr30, LrB, Lr10, Lr14a, Lr18 & 1 & 1.5 \\
\hline HGTT & Lr1, Lr2c, Lr9, Lr24, Lr26/Lr2a, Lr3a, Lr16, Lr3ka, Lr11, Lr17a, Lr30, LrB, Lr10, Lr14a, Lr18 & 1 & 1.5 \\
\hline FKTT & Lr1, Lr2a, Lr9/Lr2c, Lr3a, Lr16, Lr24, Lr26, Lr3ka, Lr11, Lr17a, Lr30, LrB, Lr10, Lr14a, Lr18 & 1 & 1.5 \\
\hline FGTT & Lr1, Lr2a, Lr9, Lr24, Lr26/Lr2c, Lr3a, Lr16, Lr3ka, Lr11, Lr17a, Lr30, LrB, Lr10, Lr14a, Lr18 & 1 & 1.5 \\
\hline Total & & 66 & 100 \\
\hline
\end{tabular}

The frequency of monopustule isolates virulent to $\operatorname{Lr} 9$ was $13 \%$, which is substantially higher than that over the last 20 $\mathrm{yr}$, the maximal frequency of virulence (10\%) was noticed in 2003 (Babayants, 2011). The virulence to $L r 29$ was $11 \%$ lower than that in previous years. Gene Lr64 was studied for the first time, the frequency of virulent monopustule isolates to it was $19 \%$. In comparison with previous years the frequency of virulence to $L r 26$ slightly decreased (17\%), in previous years it varied from $40 \%$ to $80 \%$ (Babayants, 2011). The frequency of virulent monopustule isolates to gene $\operatorname{Lr} 24$ was $26 \%$, in previous years it varied from low (less than $10 \%$ ) in 1996, 1998, 2002, 2006, 2007 to high in 1999, 2001, and 2003 (> 50\%) (Babayants, 2011).

The frequency of virulence to $L r 25$ was $26 \%$ (Table 4). Medium frequency of virulence was observed against genes $L r 2 a, L r 21, L r 22 a$ and $L r 23$. A high frequency of virulence was noticed to genes $\operatorname{LrB}, \operatorname{Lrl}, \operatorname{Lr} 2 c, \operatorname{Lr} 3 a$, Lr3ka, Lr10, Lr11, Lr14a, Lr16, Lr17, Lr18, Lr20 and Lr30 (Table 4). Virulence to lines KS86WGRC02 (Lr39/41), KS92WGRC16 (Lr42, Lr39, Lr21) and 'Pavon $753^{\prime}(\operatorname{Lr} 47)$ was $70 \%, 54 \%$, and $21 \%$ respectively, to 'Pavon 76' (Lr1, Lr10, Lr13, Lr14a, Lr2b, Lr22a, Lr27, Lr46) was 96\% (Table 5).
Effectiveness of the $\boldsymbol{L r}$-genes in the seedling stage

Seedling resistance of $L r$-genes was studied after artificial inoculation by local population of leaf rust. The effectiveness of genes was divided according to the type of reaction into the groups VR, R, MS, S, and VS. The genes within each group were subdivided also according to the disease severity (Table 6).

Highly efficient were $\operatorname{Lr} 9$ and $\operatorname{Lr} 19$, disease symptoms were not observed. Genes $L r 29$ and $L r 47$ also had resistance type of reaction, but the disease severity was $5 \%$ and $25 \%$, respectively (Table 6). Moderately susceptible were genes $L r 24$ and $L r 25$ with a disease severity of 5\%, this group also included genes $\operatorname{Lr} 56$ with a disease severity of $10 \%$, $15 \%$ for $L r 64, L r 39$ and $L r 42,25 \%$ for $L r 45,40 \%$ for $L r 15$, $L r 16, L r 51$, and $L r 52,65 \%$ for $L r 44$ and $L r 50$ (Table 6). The greater part of genes fell within the group exhibiting susceptibility. Lines carrying genes $L r 23, L r 22 a$ showed lower level of susceptibility (5\%) in this group, a bit higher level of susceptibility $(10 \%)$ was demonstrated by lines with $L r 18, L r 21$ and $L r 26$ genes (Table 6). Lines with genes $L r 1, L r 3 b g$ and 'Odesskaya polukarlikovaya' were highly susceptible with a susceptibility level of $65 \%$ (Table 6). 
Table 4. Frequency of occurrence of virulent isolates of Puccinia triticina in the south of Ukraine in 2013 (at the seedling stage).

\begin{tabular}{|c|c|c|c|}
\hline Gene & $\begin{array}{c}\text { Accession } \\
\text { number }\end{array}$ & Pedigree $^{2}$ & $\begin{array}{l}\text { Frequency of } \\
\text { virulence, \% }\end{array}$ \\
\hline $\operatorname{Lr} B$ & GSTR 446 & Thatcher*6/Brevit & 97 \\
\hline Lrl & GSTR 402 & Thatcher*6/Centenario & 91 \\
\hline $\operatorname{Lr} 2 a$ & GSTR 403 & Thatcher*6/Webster & 78 \\
\hline $\operatorname{Lr} 2 c$ & GSTR 405 & Thatcher*6/Brevit & 86 \\
\hline$L r 3 a$ & GSTR 406 & Thatcher*6/Democrat & 94 \\
\hline Lr3ka & GSTR 408 & Thatcher*6/Klein Aniversario & 84 \\
\hline $\operatorname{Lr} 9$ & GSTR 409 & Thatcher*6/Aegilops umbellulata & 13 \\
\hline $\operatorname{Lr} 10$ & GSTR 410 & Thatcher*6/Lee & 92 \\
\hline $\operatorname{Lr} 11$ & GSTR 411 & Thatcher*6/Hussar & 89 \\
\hline $\operatorname{Lr} 14 a$ & GSTR 414 & Thatcher*6/Hope & 95 \\
\hline $\operatorname{Lr} 16$ & GSTR 417 & Thatcher*6/Exchange & 93 \\
\hline $\operatorname{Lr} 17$ & GSTR 418 & Thatcher*6/Klein Lucero & 88 \\
\hline $\operatorname{Lr} 18$ & GSTR 419 & Thatcher*6/Africa 43 & 94 \\
\hline $\operatorname{Lr} 19$ & GSTR 420 & Thatcher*6/Agropyron elongatum & 0 \\
\hline $\operatorname{Lr} 20$ & GSTR 421 & Thatcher*6/Thew & 81 \\
\hline $\operatorname{Lr} 21$ & GSTR 422 & Thatcher*6/Aegilops tauschii & 68 \\
\hline $\operatorname{Lr} 22 a$ & GSTR 423 & Thatcher*6/Aegilops tauschii & 78 \\
\hline $\operatorname{Lr} 23$ & GSTR 424 & Thatcher*6/Gabo & 66 \\
\hline $\operatorname{Lr} 24$ & GSTR 425 & Thatcher*6/Agropyron elongatum & 26 \\
\hline $\operatorname{Lr} 25$ & GSTR 426 & Thatcher*6/Rosen (rye) & 45 \\
\hline $\operatorname{Lr} 26$ & GSTR 427 & Thatcher*6/Imperial (rye) & 18 \\
\hline $\operatorname{Lr} 29$ & GSTR 429 & Thatcher*6/Agropyron elongatum & 11 \\
\hline $\operatorname{Lr} 30$ & GSTR 430 & Thatcher*6/Terenzio & 92 \\
\hline Lr64 & GSTR 445 & Thatcher*6/Triticum dicoccoides & 19 \\
\hline
\end{tabular}

${ }^{1}$ Accession numbers in accordance with Research Service of Germplasm Resource Information Network (GRIN).

${ }^{2}$ Pedigree in accordance with Genetic Resources Information System for Wheat and Triticale (GRIS).

Table 5. Frequency of occurrence of virulent monopustule isolates of Puccinia triticina in cultivars/lines with $\mathbf{L r}$-genes in the south of Ukraine in 2013.

\begin{tabular}{lllcc}
\hline № п/п & Cultivar, line & \multicolumn{1}{c}{ Lr-gene ( $^{1}$} & $\begin{array}{c}\text { Accession } \\
\text { number }^{2}\end{array}$ & $\%$ \\
\hline 1 & KS86WGRC02 & Lr39 & PI 504517 & 70 \\
2 & KS92WGRC16 & Lr42, Lr39, Lr21 & PI 592728 & 54 \\
3 & Pavon 753 & Lr47 & GSTR 440 & 21 \\
4 & Pavon 76 & Lr1, Lr10,Lr13, Lr14a, & PI 519847 & 96 \\
& & Lr2b, Lr22a, Lr27, Lr46 & & \\
\hline
\end{tabular}

${ }^{1}$ The presence of $L r$-genes in accordance with Genetic Resources Information System for Wheat and Triticale (GRIS).

${ }^{2}$ Accession numbers in accordance with Research Service of Germplasm Resource Information Network (GRIN).

Table 6. Results of phytopathological evaluation of $L r$-genes at the seedling stage.

\begin{tabular}{|c|c|c|}
\hline $\begin{array}{l}\text { Type of } \\
\text { infection }\end{array}$ & $\begin{array}{l}\text { Disease } \\
\text { severity }\end{array}$ & Gene, cultivar \\
\hline VR & 0 & $\operatorname{Lr} 9, \operatorname{Lr} 19$ \\
\hline \multirow[t]{2}{*}{$\mathrm{R}$} & 5 & $\operatorname{Lr} 29$ \\
\hline & 25 & $\operatorname{Lr} 47$ \\
\hline \multirow[t]{6}{*}{ MS } & 5 & Lr24, Lr25 \\
\hline & 10 & $\operatorname{Lr} 56$ \\
\hline & 15 & Lr64, Lr39, Lr42 \\
\hline & 25 & $\operatorname{Lr} 45$ \\
\hline & 40 & Lr15, Lr16, Lr51, Lr52 \\
\hline & 65 & Lr44, Lr50 \\
\hline \multirow[t]{6}{*}{$\mathrm{S}$} & 5 & Lr23, $\operatorname{Lr} 22 a$ \\
\hline & 10 & $\operatorname{Lr} 18, \operatorname{Lr} 21, \operatorname{Lr} 26$ \\
\hline & 15 & Lr3ka, Lr20, Lr45 \\
\hline & 25 & Lr35, Lr46, Lr54, Lr63 \\
\hline & 40 & $\begin{array}{l}\operatorname{Lr} 2 a, \operatorname{Lr} 2 c, \operatorname{Lr} 10, \operatorname{Lr} 11, \operatorname{Lr} 12, \operatorname{Lr} 17, \operatorname{Lr} 27, \operatorname{Lr} 30, \operatorname{Lr} 32 \\
\operatorname{Lr} 34, \operatorname{Lr} 36, \operatorname{Lr} 37, \operatorname{Lr} 60\end{array}$ \\
\hline & 65 & Lr2b, Lr3, Lr13, Lr14a, Lr14b, Lr28, Lr33, Lr38, Lr53 \\
\hline VS & 65 & Lr1, Lr3bg, 'Odesskaya polukarlikovaya' \\
\hline
\end{tabular}

Effectiveness of the $\boldsymbol{L r}$-genes in the field

Phytopathological evaluation of wheat lines and cultivars with known genes of resistance to $P$. triticina was observed in the infected nurseries in 2013 and 2014 (Table 7).

Environmental conditions during pathogen inoculation were more favorable in 2013, indicator of disease development 'Odesskaya polukarlikovaya' scored 2 points. In 2014, there was observed a slightly lower infection pressure of the pathogen, 'Odesskaya polukarlikovaya' scored 4 points. Genes $\operatorname{Lr} 9, \operatorname{Lr} 19, \operatorname{Lr} 24$, $L r 29, L r 42, L r 47, L r 50$ and $L r 51$ were resistant in the field tests during the two experimental years. The genes $L r 25$, Lr44, Lr52, Lr53, Lr56 and Lr64 were resistant in 2014, but in 2013 they were susceptible (Table 7).

Table 7. Results of phytopathological evaluation of $\mathbf{L r}$-genes at the adult plant stage.

\begin{tabular}{|c|c|c|c|c|}
\hline Gene & Status/Name & Accession number ${ }^{1}$ & $2013^{2}$ & $2014^{2}$ \\
\hline Lrl & NIL & GSTR 402 & 2 & 4 \\
\hline $\operatorname{Lr} 2 a$ & NIL & GSTR 403 & 2 & 4 \\
\hline$L r 2 b$ & NIL & GSTR 404 & 2 & 4 \\
\hline$L r 2 c$ & NIL & GSTR 405 & 2 & 3 \\
\hline Lr3 & NIL & GSTR 406 & 2 & 2 \\
\hline Lr3bg & NIL & GSTR 407 & 2 & 4 \\
\hline Lr3ka & NIL & GSTR 408 & 2 & 5 \\
\hline $\operatorname{Lr} 9$ & NIL & GSTR 409 & 8 & 7 \\
\hline Lr10 & NIL & GSTR 410 & 2 & 4 \\
\hline Lr11 & NIL & GSTR 411 & 2 & 4 \\
\hline $\operatorname{Lr} 12$ & NIL & GSTR 412 & 6 & 5 \\
\hline Lr13 & NIL & GSTR 413 & 2 & 5 \\
\hline Lrl4a & NIL & GSTR 414 & 2 & 3 \\
\hline$L r 14 b$ & NIL & GSTR 415 & 2 & 4 \\
\hline Lr15 & NIL & GSTR 416 & 4 & 5 \\
\hline Lr16 & NIL & GSTR 417 & 2 & 5 \\
\hline $\operatorname{Lr} 17$ & NIL & GSTR 418 & 5 & 5 \\
\hline $\operatorname{Lr} 18$ & NIL & GSTR 419 & 5 & 5 \\
\hline $\operatorname{Lr} 19$ & NIL & GSTR 420 & 9 & 7 \\
\hline $\operatorname{Lr} 20$ & NIL & GSTR 421 & 2 & 6 \\
\hline $\operatorname{Lr} 21$ & NIL & GSTR 422 & 5 & 4 \\
\hline$L r 22 a$ & NIL & GSTR 423 & 6 & 4 \\
\hline $\operatorname{Lr} 23$ & NIL & GSTR 424 & 6 & 5 \\
\hline$L r 24$ & NIL & GSTR 425 & 8 & 7 \\
\hline$L r 25$ & NIL & GSTR 426 & 5 & 8 \\
\hline $\operatorname{Lr} 26$ & NIL & GSTR 427 & 4 & 6 \\
\hline $\operatorname{Lr} 27$ & Gatcher & PI 377884 & 3 & 5 \\
\hline $\operatorname{Lr} 28$ & NIL & GSTR 428 & 4 & 5 \\
\hline $\operatorname{Lr} 29$ & NIL & GSTR 429 & 7 & 8 \\
\hline Lr30 & NIL & GSTR 430 & 2 & 5 \\
\hline Lr32 & NIL & GSTR 431 & 4 & 5 \\
\hline Lr33 & NIL & GSTR 432 & 2 & 3 \\
\hline Lr34 & NIL & GSTR 433 & 5 & 5 \\
\hline Lr35 & NIL & GSTR 434 & 5 & 5 \\
\hline Lr36 & Genetic material & GSTR 435 & 6 & 6 \\
\hline Lr37 & NIL & GSTR 436 & 5 & 6 \\
\hline $\operatorname{Lr} 38$ & NIL & GSTR 437 & 2 & 4 \\
\hline Lr39/41 & KS86WGRC02 & PI 504517 & 6 & 7 \\
\hline Lr42 & KS92WGRC16 & PI 592728 & 9 & 8 \\
\hline Lr44 & NIL & GSTR 438 & 4 & 8 \\
\hline Lr45 & NIL & GSTR 439 & 6 & 5 \\
\hline$L r 46$ & Pavon 76 & PI 519847 & 6 & 6 \\
\hline Lr47 & Pavon 753 & GSTR 440 & 8 & 8 \\
\hline $\operatorname{Lr} 50$ & KS96WGRC36 & PI 604221 & 9 & 8 \\
\hline Lr51 & NIL & GSTR 441 & 8 & 8 \\
\hline $\operatorname{Lr} 52$ & NIL & GSTR 442 & 6 & 8 \\
\hline Lr53 & $98 \mathrm{M} 71$ & PI 648417 & 6 & 8 \\
\hline Lr54 & Genetic material & PI 648418 & 3 & 6 \\
\hline Lr56 & Genetic material & PI 648419 & 8 & 8 \\
\hline Lr60 & Genetic material & GSTR 443 & 3 & 4 \\
\hline Lr63 & NIL & GSTR 444 & 2 & 4 \\
\hline Lr64 & NIL & GSTR 445 & 6 & 8 \\
\hline \multicolumn{3}{|c|}{ 'Odesskaya polukarlikovaya' indicator of disease development } & 2 & 4 \\
\hline
\end{tabular}




\section{DISCUSSION}

Twelve phenotypes were identified on the old differential set in the population of leaf rust in 2013. The most common phenotypes were $77(75 \%)$ and $144(6 \%)$. Over the last 20 yr a total of 47 known and 15 new races in the population of P. triticina were found in the south of Ukraine (Babayants, 2011). According to the North American nomenclature, the dominant phenotypes in the population of leaf rust were TGTT (24\%) and TJTT (8\%), both phenotypes possess a broad spectrum of virulence and according to the old nomenclature both may belong to the "race 77". However, the first phenotype was avirulent to $L r 24$, whereas the second phenotype was virulent to it. The obtained results showed that the North American differential set provides more important information and much better differential ability than the old standard differential set used before in Ukraine. The presence of the effective gene $\operatorname{Lr} 9$ and genes with partial resistance $L r 24$ and $L r 26$ in the nomenclature makes more significant phenotype differentiation for south of Ukraine. The advantage of the North American nomenclature over the standard differential set also was established by other researchers (Todorova and Kiryakova, 2001). Moreover, the researchers who work independently using the letter code nomenclature will give the same codename to any new physiologic pathotypes that code the same on this system. This allows researchers to speak the same language and have a common understanding of the virulence phenotypes. However, some $L r$-genes which are effective in the south of Ukraine are not included in the differential set. Therefore for virulence analysis we used additional 'Thatcher' near-isogenic lines with $\operatorname{Lr} 19, \operatorname{Lr} 20$, Lr21, Lr22a, Lr23, Lr24, Lr25, Lr29 and Lr64 Lr-genes, 'Pavon 76' (Lr1, Lr10, Lr13, Lr14a, Lr2b, Lr22a, Lr27, $L r 46)$ and wheat lines 'Pavon 753' (Lr47), KS86WGRC02 (Lr39) and KS92WGRC16 (Lr42, Lr39, Lr21) which are new or possess resistance and present local interest for our environments. Virulence analysis exhibited that the frequency of virulence to $\operatorname{Lr} 9$ was increased by $13 \%$ in 2013. It is generally accepted, that when the frequency of virulence reaches higher than $10 \%$ efficiency of gene may be overcome by pathogen. When the frequency of virulent pathotypes and/or their aggressiveness increase, this gene may be shortly overcome by the pathogen in the south of Ukraine. Breaking down of $\operatorname{Lr} 9$ was noticed in the USA and Canada (McCallum et al., 2011; Kolmer et al., 2012).

Lr19 remains highly efficient, no virulent isolates were found in the population of leaf rust. Virulence to that resistant gene has not been found in other European countries (Mesterházy et al., 2000; Huerta-Espino et al., 2011). But according to the published data a large quantity of virulent pathotypes (40\%) were detected in the population of leaf rust in Volga region of Russia (Kurbanova, 2011; Ivanova, 2013).

The gene Lr24 derived from Agropyron elongatum (Host.) Neviski (McIntosh et al., 2013). According to the last monitoring, virulence to $\operatorname{Lr} 24$ was very rare in Germany, Spain, Hungary, Slovak Republic (HuertaEspino et al., 2011), and Czech Republic (Hanzalova et al., 2013), Lithuania (Liatukas, 2003) and China (Liu and Chen, 2012). In Ukraine, Lr24 exhibited moderate susceptibility at the seedling stage and resistance at adult plant stages.

Gene Lr29 derived from A. elongatum (McIntosh et al., 2013) under artificial inoculation by population of leaf rust at the seedling stage provided resistance; the frequency of virulence to it was $11 \%$. However, in the previous years virulence to it was relatively high. This indicates that this gene is unreliable against leaf rust (Babayants et al., 2004; Babayants, 2011).

Slow rusting gene $L r 34$ has been providing resistance for more than $50 \mathrm{yr}$, and $L r 46$ has remained effective since 1976 in the USA. Also, it was established that Lr34 has the ability to enhance the resistance to P. triticina in combination with other $L r$-genes and to provide positive pleiotropic effect to other wheat diseases (German and Kolmer, 1992; Kolmer et al., 2008). The synergy between $L r 34$ and other $L r$-genes also was detected in our environments (unpublished data). However, Lr34 in single use did not provide sufficient level of resistance in the population of southern Ukraine. It was highly susceptible at the seedling stage and susceptible (scored 6) at the adult plant stages. Gene $L r 46$ in combination with other genes Lr1, Lr10, Lr13, Lr14a, Lr2b, Lr22a, and Lr27 in background of 'Pavon 76' also did not provide sufficient level of resistance.

Gene Lr42 derived from Aegilops tauschii Coss. (McIntosh et al., 2013) exhibited moderate susceptibility in the seedling test and resistance in the field test. This indicates that it provides sufficient level of resistance only at the adult plant stages. Gene $\mathrm{Lr} 47$ derived from Triticum speltoides (Tausch) Gren. ex K. Richt. (McIntosh et al., 2013) provided resistance in seedling test and in the field in 2013 and 2014, but virulence to it at the seedling plant stage was $21 \%$. This indicates that resistance may be soon overcome by pathogen. Genes Lr50, Lr51 and Lr56 were studied by us for the first time and exhibited resistance in the field test during $2 \mathrm{yr}$, but $L r 50$ was susceptible, and Lr51, Lr56 were moderately susceptible in the seedling test. That may indicate that they act as the adult plant stages genes. Gene Lr64 derived from Triticum dicoccoides (McIntosh et al., 2013) was also studied by us for the first time. At the seedling stage plants showed moderate susceptibility, the frequency of virulence was $19 \%$, at the adult plant stages this gene provided susceptibility in 2013 and resistance in 2014. The resistance in 2014 and susceptibility in 2013 of genes $\operatorname{Lr} 25, \operatorname{Lr} 44, \operatorname{Lr} 52, \operatorname{Lr} 53$, Lr56 and Lr64 in the field test can be caused by different pathogen development. Artificial leaf rust inoculation was more successful in 2013, which can be explained by a very small amount of precipitation in April 2014 during the crucial period for inoculation (Table 1). Urediniospores 
begin to develop a germ tube and penetrate the cell only when the moisture is present in the form of dew or light rain on the leaf surface. Germination occurs after $8 \mathrm{~h}$ at 18 ${ }^{\circ} \mathrm{C}$, spores possess the ability to retain viability only $1-3 \mathrm{~d}$ after inoculation under field conditions in the absence of the dew period (Babayants and Babayants, 2014). Due the better environment conditions for pathogen development, the infection of leaf rust developed better in 2013 than in 2014. In 2013, when infection pressure was higher genes Lr25, Lr44, Lr52, Lr53, Lr56, and Lr64 were susceptible, which may indicate their insufficiency; under optimal conditions for pathogen development they cannot provide sufficient level of resistance.

The Ukrainian population of leaf rust consists of broad range of pathotypes with different spectrum and frequency of virulence. This point to high pathogen evolutionary ability, and consequently a large part of known Lrgenes have lost their efficiency and cannot be used as donors of resistance. Thus, single use of these $L r$-genes cannot provide durable defense against wheat leaf rust. Theoretically, if resistance is controlled at one single locus, only one mutation in the corresponding avirulent gene may lead to emergence of a new virulent pathotype. Therefore several breeding strategies, pyramiding and slow rusting to enhance the durability of resistance to leaf rust have been proposed. Gene pyramiding is incorporation of several $L r$-genes into a single genotype. Slow rusting is conferred by genes which provide a longer latent period of disease, lower spore production and as a result smaller areas under the disease progress curve than a susceptible control (Singh et al., 2011). For gene pyramiding it is needed to choose the major genes to which corresponds a very low ratio of virulence in pathogen population and then they could be used in combination with seedling partial resistance genes or in combination with adult non-specific genes (Krattinger et al., 2009; Dakouri et al., 2013).

\section{CONCLUSIONS}

The population of leaf rust consisted of different phenotypes in the south of Ukraine. According to the standard differential set, there were identified 12 phenotypes of which the most common were $77(75 \%)$ and $144(6 \%), 149(4 \%), 117(3 \%), 192(2 \%), 114(2 \%)$, $6(2 \%), 21$ (2\%), $42(1 \%), 57(1 \%), 20(1 \%), 122(1 \%)$. The North American nomenclature provides much more differential ability, and using this nomenclature we identified 40 phenotypes, of which the most frequent were TGTT (24\%) and TJTT (8\%), phenotypes TRTT (1.5\%) and TSTT $(1.5 \%)$ possess the broadest spectrum of virulence among the isolates found in the south of Ukraine. Among all studied $L r$-genes no isolates were virulent to $L r 19$, against other $L r$-genes virulence frequency varied from 11 to $97 \%$. Low frequency of virulence was observed to $\operatorname{Lr} 29$ (11\%), $\operatorname{Lr} 9(13 \%)$ and $\operatorname{Lr} 47(21 \%)$, high level of virulence was detected to other genes. Most of the known $L r$-genes do not provide resistance either at the seedling or at the adult plant stage. Genes $\operatorname{Lr} 9, \operatorname{Lr} 19, \operatorname{Lr} 29$ and $L r 47$ were highly effective both at the seedling stage and in the field test. Genes Lr24, Lr42, Lr50, Lr51 and Lr56 were effective only at the adult plant stage during the two experimental years.

\section{LITERATURE CITED}

Babayants, O.V. 2011. Immunological characteristic of wheat plant resources and verification of genetic protection against pathogens of fungal aetiology in the Steppe region of Ukraine. $\mathrm{PhD}$ thesis. Plant Breeding and Genetics Institute, Kyiv, Ukraine (in Ukrainian).

Babayants, O.V., and L.T. Babayants. 2014. Base principals and methodology of phytopathological estimation of wheat resistance to diseases. 401 p. Plant Breeding and Genetics Institute, National Center of Seed and Cultivar Investigation (PBGI-NC SCI), Odessa, Ukraine (in Russian).

Babayants, L.T., A.A. Vasiliev, V.A. Traskovetskaya, and O.V. Babayants. 2004. Race composition of Puccinia recondita f. sp. tritici in the south of Ukraine and resistance of wheat varieties. Collection of papers. Plant Breeding and Genetics Institute 6:279288 (in Russian).

Dakouri, A., B.D. McCallum, N. Radovanovic, and S. Cloutier. 2013. Molecular and phenotypic characterization of seedling and adult plant leaf rust resistance in a world wheat collection. Molecular Breeding 32:663-677. doi:10.1007/s11032-013-9899-8.

German, S.E., and J.A. Kolmer. 1992. Effect of gene Lr34 in the enhancement of resistance to leaf rust of wheat. Theoretical and Applied Genetics 84:97-105. doi:10.1007/BF00223987.

Goyeau, H., R. Park, B. Schaeffer, and C. Lannou. 2006. Distribution of pathotypes with regard to host cultivars in French wheat leaf rust populations. Phytopathology 96:264-273. doi:10.1094/ PHYTO-96-0264.

GRIS. 2014. Genetic Resources Information System for Wheat and Triticale (GRIS) [Online database]. Available at http:// wheatpedigree.net (accessed November 2014).

Hanzalova, A., and P. Bartos. 2006. Physiologic specialization of wheat leaf rust (Puccinia triticina Eriks.) in the Czech Republic in 2001-2004. Czech Journal of Genetics and Plant Breeding 42:126-131.

Hanzalova, A., P., Bartos, and T. Sumikova. 2013. Physiological specialization of wheat leaf rust (Puccinia triticina Eriks.) in the Czech Republic in 2009-2011. Czech Journal of Genetics and Plant Breeding 49:103-108.

Huerta-Espino, J., R.P. Singh, S. German, B.D. McCallum, R.F. Park, W.Q. Chen, et al. 2011. Global status of wheat leaf rust caused by Puccinia triticina. Euphytica 179:143-160. doi:10.1007/s10681011-0361-x.

Ivanova, O.V. 2013. The sources of spring wheat resistance to leaf rust and variability of population composition in the Volga region. $\mathrm{PhD}$ thesis. Saratov State Vavilov Agrarian University, Saratov, Russia (in Russian).

Johnson, C.O., and L.E. Browder. 1966. Seventh revision of the international register of physiologic races of Puccinia recondita f.sp. tritici. Plant Disease Reporter 50:756-760.

Kolmer, J.A. 2005. Tracking wheat rust on a continental scale. Current Opinion in Plant Biology 8:441-449. doi:10.1016/j. pbi.2005.05.001.

Kolmer, J.A., and M.E. Hughes. 2013. Physiologic specialization of Puccinia triticina on wheat in the United States in 2011. Plant Disease 97:1103-1108. doi:10.1094/PDIS-11-12-1068-SR.

Kolmer, J.A., and J.Q. Liu. 2000. Virulence and molecular polymorphism in international collections of the wheat leaf rust fungus Puccinia triticina. Phytopathology 90:427-436. doi:10.1094/PHYTO.2000.90.4.427. 
Kolmer, J.A., D.L. Long, and M.E. Hughes. 2007. Physiologic specialization of Puccinia triticina on wheat in the United States in 2005. Plant Disease 91:979-984. doi:10.1094/PDIS-91-8-0979.

Kolmer, J.A., D.L. Long, and M.E. Hughes. 2012. Physiologic specialization of Puccinia triticina on wheat in the United States in 2010. Plant Disease 96:1216-1221. doi:10.1094/PDIS-01-120048-SR.

Kolmer, J.A., R.P. Singh, D.F. Garvin, L. Viccars, H.M. William, J. Huerta-Espino, et al. 2008. Analysis of the $L r 34 / Y r 18$ rust resistance region in wheat germplasm. Crop Science 48:18411852. doi:10.2135/cropsci2007.08.0474.

Krattinger, S.G., E.S. Lagudah, W. Spielmeyer, R.P. Singh, J. HuertaEspino, H. McFadden, et al. 2009. A putative ABC transporter confers durable resistance to multiple fungal pathogens in wheat. Science 323:1360-1363. doi:10.1126/science.1166453.

Kurbanova, P.M. 2011. Genetic diversity of soft spring wheat with respect to efficiency of adult leaf rust resistance. $\mathrm{PhD}$ thesis. N.I. Vavilov Research Institute of Plant Industry, St. Petersburg, Russia (in Russian).

Liatukas, Ž. 2003. Virulence of isolates of the winter wheat leaf rust. Biologija 49:77-80.

Liu, T.G., and W.Q. Chen. 2012. Race and virulence dynamics of Puccinia triticina in China during 2000-2006. Plant Disease 96:1601-1607. doi.org/10.1094/PDIS-06-10-0460-RE.

Long, D.L., and J.A. Kolmer. 1989. A North American System of Nomenclature for Puccinia triticina. Phytopathology 79:525-529. doi:10.1094/Phyto-79-525.

Mains, E.B., and H.S. Jackson. 1926. Physiologic specialization in the leaf rust of wheat, Puccinia triticina Erikss. Phytopathology 16:89-119.

Mantovani, P., M. Maccaferri, R. Tuberosa, and J.A. Kolmer. 2010. Virulence phenotypes and molecular genotypes in collections of Puccinia triticina from Italy. Plant Disease 94:420-424. doi:10.1094/PDIS-94-4-0420.

McCallum, B.D., P. Seto-Goh, and A. Xue. 2011. Physiologic specialization of Puccinia triticina, the causal agent of wheat leaf rust, in Canada in 2008. Canadian Journal of Plant Pathology 33:541-549. doi:10.1080/07060661.2011.627950.
McIntosh, R.A., C.R. Wellings, and R.F. Park. 1995. Wheat rust. An atlas of resistance genes. CSIRO, Dickson, Canberra, Australia.

McIntosh, R.A., Y. Yamazaki, J. Dubcovsky, W.J. Rogers, C. Morris, R. Appels, et al. 2013. Catalogue of gene symbols for wheat. In KOMUGI Integrated Wheat Science Database. Available at http://www.shigen.nig.ac.jp/wheat/komugi/genes/download.jsp (accessed November 2014).

McVey, D.V., M. Nazim, K.J. Leonard, and D.L. Long. 2004. Patterns of virulence diversity in Puccinia triticina on wheat in Egypt and the United States in 1998-2000. Plant Disease 88:271279. doi:10.1094/PDIS.2004.88.3.271.

Mesterházy, A., P. Bartoš, H. Goyeau, R. Niks, M. Csösz, O. Andersen, et al. 2000. European virulence survey for leaf rust in wheat. Agronomie 20:793-804. doi:10.1051/agro:2000104.

Singh, R.P. 1991. Pathogenicity variations of Puccinia recondita f. sp. tritici and $P$. graminis f. sp. tritici in wheat growing areas of Mexico during 1988 and 1989. Plant Disease 75:790-794. doi:10.1094/PD-75-0790.

Singh, R.P., J. Huerta-Espino, S. Bhavani, S.A. Herrera-Foessel, D. Singh, P.K. Singh, et al. 2011. Race non-specific resistance to rust diseases in CIMMYT spring wheats. Euphytica 179:175-186. doi:10.1007/s10681-010-0322-9.

Terefe, T.G., B. Visser, L. Herselman, R. Prins, T. Negussie, J.A. Kolmer, et al. 2014. Diversity in Puccinia triticina detected on wheat from 2008 to 2010 and the impact of new races on South African wheat germplasm. European Journal of Plant Pathology 139:95-105. doi:10.1007/s10658-013-0368-3.

Todorova, M., and V. Kiryakova. 2001. Physiologic specialization of Puccinia recondita f. sp. tritici in Bulgaria in 1999. Cereal Rust and Powdery Mildews Bulletin. Available at http://www.crpmb. org/2001/0126todovora/ (accessed November 2014).

USDA ARS. 2014. National Genetic Resources Program. Germplasm Resources Information Network (GRIN) [Online Database]. United States Department of Agriculture (USDA), Agricultural Research Service (ARS), National Germplasm Resources Laboratory, Beltsville, Maryland. Available at http:// www.ars-grin.gov (accessed November 2014). 